\title{
Germanica
}

\section{Échanges et transferts entre Zagreb (Agram) et Vienne autour de 1900}

\section{Fridrun Rinner}

\section{(2) OpenEdition}

\section{Journals}

Édition électronique

URL : http://journals.openedition.org/germanica/567

DOI : 10.4000/germanica.567

ISSN : 2107-0784

Éditeur

Université de Lille

Édition imprimée

Date de publication : 1 décembre 2008

Pagination : 115-122

ISBN : 978-2-913857-22-6

ISSN : 0984-2632

Référence électronique

Fridrun Rinner, «Échanges et transferts entre Zagreb (Agram) et Vienne autour de 1900 », Germanica [En ligne], 43 | 2008, mis en ligne le 01 décembre 2010, consulté le 06 octobre 2020. URL : http:// journals.openedition.org/germanica/567 ; DOI : https://doi.org/10.4000/germanica.567

Ce document a été généré automatiquement le 6 octobre 2020.

(c) Tous droits réservés 


\title{
Échanges et transferts entre Zagreb (Agram) et Vienne autour de 1900
}

\author{
Fridrun Rinner
}

1 Les échanges entre Vienne et Zagreb sont l'expression d'un processus d'individualisation culturelle et politique croate qui a commencé avec la « renaissance nationale » des années 1830 et qui a été l'objet de discussions à l'université de Zagreb autour de 1900. Si ce mouvement de renaissance nationale commença par se manifester dans la littérature et dans la langue littéraire, c'est à partir de 1848 et surtout du compromis austro-hongrois en 1868 que l'on chercha aussi à réaliser cette renaissance dans le domaine politique: il s'agissait alors d'unir les pays croates (kroatische Staatsländer), d'aboutir à l'indépendance de l'État croate et à la formation d'une identité culturelle et politique nationale du peuple croate. Les recherches systématiques sur son propre passé sont liées à l'université où l'on s'interrogeait sur les influences exercées par les "grandes philosophies nationales" européennes sur l'évolution de la philosophie croate. Une philosophie nationale croate - pensait-on - pourrait aider à renforcer la conscience nationale.

2 C'est en 1874, lors de l'inauguration de la nouvelle université de Zagreb, que son premier président, Matija Mesić (1826-1878) insista dans son discours inaugural sur le devoir humain et national des scientifiques de respecter la particularité du peuple, sa vision du monde et ses conditions de vie ${ }^{1}$.

3 C'est sur la base d'un tel programme de "culture nationale » qu'il faut également considérer le développement de la philosophie dans cette «nouvelle université nationale » à la fin $\mathrm{du} \mathrm{xIX}^{\mathrm{e}}$ siècle. Certes, il existait déjà des liens étroits avec la philosophie viennoise, voire autrichienne avant l'ouverture de cette "nouvelle université ». C'est en 1527 que le Parlement croate avait élu Ferdinand I ${ }^{\text {er }}$ (1503-1564) roi et avait ainsi intégré le pays dans la Monarchie habsbourgeoise. En 1607, les jésuites avaient ouvert le premier lycée de Zagreb, et trente ans plus tard, on créa la première "Faculté de théologie ». Par la suite, les deux furent réunis dans l'Academia Zagrabiensis estimée parmi les académies jésuites de la Monarchie, à tel point que l'empereur Léopold I ${ }^{\mathrm{er}}$ (1640-1705) transforma en 1669 cette académie en une université. Celle-ci 
fut transformée en 1776 en « Académie royale des sciences » avec tous les privilèges qui étaient accordés aux universités du Reich allemand et de ses Erbländer » (entre autres Cologne, Mayence, Prague, Graz, Koshice etc). Le jésuite Josip Ruder Bošković (1711-1787), philosophe et scientifique, avait fortement marqué les liens entre les philosophies croate et viennoise en introduisant dans toutes les universités de la Monarchie la réception des théories de Newton ${ }^{2}$. La réforme éclairée de Marie-Thérèse, introduite dans toutes les universités de la Monarchie, donc aussi dans l'Academia Zagrebiensis, conduisit finalement à un éclectisme philosophique qu'on peut constater entre autres dans l'œuvre Philosophia critice elaborata ${ }^{3}$ de Simon Čučić (1784-1828).

Il faut également mentionner l'influence considérable de Johann Friedrich Herbart (1776-1841) et la présence de "l'herbartisme » dans les manuels de philosophie, de psychologie et de pédagogie en Croatie dans la deuxième moitié du XIX ${ }^{e}$ siècle ${ }^{4}$.

D'après les recherches de Franjo Zenko, une dizaine d'étudiants croates faisaient autour de 1900 leurs études de philosophie à Vienne et obtinrent leur diplôme de docteur dans cette université. Le plus connu d'entre eux était Franjo Marković (1845-1915), étudiant du philosophe Robert Zimmermann (1824-1898), professeur à l'université de Vienne. Marković représentait vers la fin du siècle la figure clé de la philosophie croate. Nommé en 1874 professeur à l'université de Zagreb, il joua le même rôle dans son université que son maître à Vienne. Marković se fondait - comme Zimmermann - sur les théories de Herbart et considérait que la mission de la philosophie était de soutenir le peuple croate dans sa quête d'indépendance culturelle et politique. Il voulait aussi assurer l'autonomie de la philosophie nationale par rapport aux grands courants philosophiques européens et tenait surtout à se distancier de l'influence germanique, plus précisément de l'idéalisme allemand. Marković cherchait à associer la «nouvelle philosophie » aux racines de la philosophie croate (au-delà de la césure kantienne et idéaliste), antérieure à la Renaissance, c'est-à-dire qu'il cherchait à la reconstituer telle qu'elle existait à ses débuts au Moyen Âge.

6 Après ce renouveau de la philosophie scolaire (Schulphilosophie) à l'université de Zagreb, la philosophie avait toute sa place, à côté de la littérature, de la linguistique et de l'histoire dans l'affirmation nationale du peuple croate. La philosophie faisait donc partie du programme culturel du peuple (Volkswesen) - un rôle identique à celui que Zimmermann lui avait accordé à Vienne.

7 Les théories de Herbart se retrouvaient aussi dans le domaine de la pédagogie où l'éducation et l'instruction ne devaient pas être conçues séparément. D'après Herbart, l'éducateur devait d'abord dresser une sorte de carte géographique de l'esprit de chaque enfant en identifiant les différents groupes d'idées qui constituent sa pensée, pour ensuite le former en assimilant ces divers groupes à une "représentation esthétique du monde " plus cohérente. Il devrait ainsi selon lui - tout en tenant compte des dispositions physiques et du tempérament particulier de chaque enfant - l'élever de son état d'individualité à un état d'universalité. Herbart se réfère à cet égard à la culture de la Grèce antique (entre autres à l'Ulysse d'Homère).

8 Cette théorie de Herbart a rencontré un très grand succès en Europe Centrale, parce qu'elle comportait une libération et une sécularisation de la formation scolaire qui étaient nécessaires à la création d'une conscience nationale. Il faut rappeler qu'en Croatie, la recherche et l'enseignement de la philosophie étaient jusqu'à la fin du siècle aux mains des Jésuites et des Franciscains. Ce ne fut qu'en 1874 que François-Joseph I ${ }^{\text {er }}$ ordonna la séparation de l'Église et de l'école. Suite à cette sécularisation de 
l'enseignement, les maîtres durent recevoir une formation particulière pour assurer l'harmonie des forces physiques et ainsi contribuer à l'organisation d'une unité organique du corps et de l'âme du peuple croate. La première association des maîtres (Lehrerverein) fut créée en 1871 à Zagreb, et les Volkshochschulen inspirées par Friedrich Jodl pour se libérer de l'oppression exercée par certains peuples plus forts, se répandirent dans le pays. On tenta même d'établir une union en matière d'enseignement avec les autres Slaves du sud pour prouver son appartenance à une culture commune ${ }^{5}$.

Vladimir Dvorniković, un autre grand nom de la philosophie croate, a également fait ses études à Vienne et $\mathrm{y}$ a écrit sa thèse de doctorat sous la direction de Friedrich Jodl, qui défendait la nécessité d'une justification psychologique de la théorie de la connaissance (Erkenntnistheorie). En 1916, il publia un article sous le titre "Stérilité de la méthode transcendantale dans la philosophie moderne », dans lequel il mettait l'accent sur le fait que les «petits peuples » comme les Croates avaient l'avantage de «pouvoir être objectifs » par rapport aux tendances et oppositions auxquelles étaient soumis les grands peuples civilisés (il mentionnait entre autres l'empirisme unilatéral des Anglais et le kantianisme conservateur des Allemands). Dvorniković faisant le portrait du professeur viennois Adolf Stöhr, le présentait comme le psychologue par excellence, tandis qu'il jugeait son compatriote Stjepan Matičević comme " antipsychologue » (ce dernier était surtout devenu célèbre dans le domaine de la pédagogie philosophique).

On peut donc constater que l'influence de l'école viennoise de philosophie était multiple et diverse et que les disciples croates ont « importé » les controverses de leurs professeurs viennois dans leur pays.

11 Si l'on se tourne vers la littérature autour de 1900, et particulièrement vers le modernisme, on retrouve également une multiplicité de styles et de formes poétiques, en particulier pendant la période 1895-1914. La fin de la modernité croate coïncida avec le début de la Première Guerre mondiale, tandis que son début fut marqué par un événement important pour l'histoire nationale de la Croatie. C'est le 14 octobre 1895 qu'eut lieu, en présence de l'empereur François-Joseph $\mathrm{I}^{\mathrm{er}}$ et du premier ministre Bánffy, l'ouverture du nouveau théâtre national de Zagreb. Le banus croate KhuenHédérvary, d'origine hongroise, avait fait apporter du sable hongrois pour que l'empereur ne soit pas « obligé » de marcher sur le sol croate. Il s'agissait bien sûr d'une provocation qui devait montrer que les Croates étaient tenus de se soumettre au Compromis entre les Autrichiens et les Hongrois. Deux jours après cet événement, il y eut des manifestations anti-hongroises à Zagreb, des étudiants brûlèrent le drapeau hongrois. Ceux-ci furent par la suite expulsés de l'université de Zagreb et contraints de poursuivre leurs études hors de Croatie. Se formèrent ensuite deux groupes : ceux qui partaient pour Vienne et qu'on nommait les « esthètes » en raison de leur orientation particulière vers l'art, l'esthétisme étant alors en vogue dans la capitale de la Monarchie. Ce furent eux qui - après leur retour dans leur pays - dictèrent les tendances nouvelles de la littérature croate au tournant du siècle. L'autre groupe, les progressistes, poursuivit leurs études à Prague et, sous l'influence de Tomaš G. Massarýk, utilisa la littérature à des fins sociales et politiques.

12 À Vienne, les étudiants croates bannis se trouvèrent confrontés à des orientations artistiques nouvelles, une sensibilité particulière, l'expression du goût européen " fin de siècle » et importèrent ces nouvelles tendances dans la culture croate. Grâce à eux, 
la littérature croate renoua - après avoir été isolée lors de la renaissance nationale avec l'esprit européen et sortit de son cloisonnement provincialiste.

Dans le domaine littéraire, l'événement magistral au sein du groupe viennois fut la création de la revue Mladost ( Jeunesse ») en 1898. Cette " revue de littérature et d'art moderne » - tel était son sous-titre - était éditée à Vienne, mais imprimée à Zagreb. Dans sa présentation, la revue ressemblait beaucoup à la revue Ver Sacrum de la Sécession viennoise, également créée en 1898. La revue Mladost, très proche du milieu moderniste et décadent de Vienne, faisait des comptes rendus réguliers de la vie culturelle de la capitale de la Monarchie, donnait régulièrement des informations sur les concerts et expositions à Vienne, publiait des traductions et des textes d'Arthur Schnitzler ainsi que des débats autour des thèses de Hermann Bahr.

Le Zagreb artistique donnait presque l'image d'une petite Vienne (en 1900 on comptait environ, 61000 habitants), les cafés faisaient figure de centres de la vie culturelle, on y trouvait une multitude de revues, de cercles littéraires. Pour prouver qu'on était ouvert aux idées les plus modernes de l'Europe, on y publiait des articles sur la Sécession, sur les caractéristiques magiques de l'âme, sur la décadence, la télépathie, le mouvement des femmes. Le parallélisme que l'on constatait entre les évolutions à Vienne et à Zagreb à la fin du XIX siècle était donc frappant: de la même façon qu'à Vienne, les jeunes artistes étaient à l'origine de la "Sécession", avaient quitté l'association des artistes et créé «l'association autrichienne des arts plastiques ", les jeunes artistes, à Zagreb, se détournaient également du Kunstverein et avaient fondé un club indépendant de l'association d'artistes avec un programme pratiquement identique à celui de Vienne.

Cet échange culturel entre Vienne et Zagreb fut une source d'inspiration et de renouveau non seulement dans la littérature, mais aussi dans les domaines de la peinture, de la sculpture, de la musique, de l'architecture, entre autres, de sorte que l'on a pu parler d'un "retour de la culture croate vers l'Europe ». L'ouverture de la première grande exposition des sculpteurs croates en décembre 1898 était également un signe manifeste de cette orientation moderne. Le personnage le plus marquant de cet événement était sans doute Milivoj Dežman Ivanov qu'on nommait souvent le «Bahr croate » en raison de son talent d'organisateur (et de la médiocrité de ses talents littéraires). Milivoj Dežman créa ensuite la revue Hravatski salon qui publiait des reproductions d'objets exposés ainsi que des textes courts, imprimés dans un style symboliste, décadent, presque identique dans sa présentation à la revue Ver sacrum de la Sécession. Dežman y publiait aussi son manifeste «naša težnje » (= " Nos requêtes ») dans lequel il exigeait, au nom de la génération des jeunes artistes, la liberté totale de l'art, prônait le culte de l'individualisme pour dépasser le naturalisme. Comme à Vienne, cette publication fit scandale en Croatie.

Les journaux publièrent également les polémiques qui opposaient "anciens » et «modernes». Parmi les critiques des liens qu'entretenaient ces artistes avec Vienne, on trouvait Gustav Anton Matoš (1873-1914), qui se moquait de l'esthétique des capucins, parlait d'une esthétique du dilettantisme, du superficiel et de l'ignorance, lui reprochait son caractère éclectique et, comme l'autre grand poète croate Miroslav Krleža (1893-1981), il critiquait fortement Hermann Bahr, le qualifiant de "grammophone des impressions étrangères ". Matoš se tourna vers Paris pour se ressourcer, Vienne lui semblant trop médiocre. 
17 Le modernisme en Croatie avait ainsi déclenché une révolte contre les institutions existantes, contre les formes d'organisation traditionnelles, contre l'art académique et l'historicisme. Bien que Mladost suscitât un grand intérêt parmi les jeunes en Croatie, cette revue provoqua aussi maintes discussions et polémiques parmi les cercles puritains croates, qui s'opposèrent à cette décadence et à ce cosmopolitisme "viennois " qui exerçait une influence néfaste sur la culture moderne à Zagreb. L'un des griefs était l'insuffisante mise en valeur du terroir croate, mais aussi de la tradition, de la littérature et, plus encore, de la société et de l'église croates. Dans un article paru dans Mlada Hrvatska, Arthur Grado décrit la décadence et le snobisme comme une importation malheureuse venue d'Europe qui n'avait aucun lien avec le sol et la tradition croates $^{6}$. Il considérait les cafés comme des lieux de rendez-vous de suicidaires qui - par leur consommation de café, de tabac et de livres - se détruisaient, à ses yeux, le cerveau? ${ }^{7}$ Il voyait la « jeune Croatie " comme une légion de rêveurs qui erraient dans le monde et regrettait la perte de cette énergie vitale. Ante Tresić Pavičić, de son côté, jugeait les discussions dans les cafés très intéressantes, mais estimait aussi que la jeune intelligentia croate « sans perspective » y perdait son temps et représentait, pour leur pays, des « hommes perdus».

18 Milutin Cihlar Nahajev, un autre ancien étudiant à Vienne, fit de la capitale autrichienne, dans sa nouvelle La grande ville, un héros négatif, en opposition totale au village, à la nature; Vienne figurait comme l'antithèse d'une Croatie idyllique, car les écrivains modernistes croates adoptaient les idées de la Wiener Moderne, considérant l'existence du monde comme un phénomène esthétique, le paradigme esthétique comme primat de l'art sur la réalité.

19 De tous temp, l'image de Vienne dans la littérature croate suscita à la fois fascination et rejet. Depuis le xvi siècle, la Croatie fait partie de la Monarchie, et il n'est donc pas étonnant que Vienne soit présente dans la littérature croate. La capitale de la Monarchie est décrite comme un foyer du vice et de la décadence morale (August Šenoa, 1838-1881, Eugen Kumičić, 1850-1904). Dans le roman de Josip Eugen Tomić (1843-1906) Pour le roi - pour la patrie, Vienne est présentée comme le lieu des aventures amoureuses, mais aussi comme centre de la modernité, comme une ville cosmopolite, lieu de rencontre des aristocrates et des artistes.

20 Les modernistes, pour qui Vienne a été une source d'inspiration à bien des égards, ont donc produit une image négative de cette ville attrayante, mais en même temps répugnante, dangereuse et destructrice. Les raisons de ce refus étaient probablement d'ordre politique et témoignent d'un ressentiment vis-à-vis de la Monarchie austrohongroise.

21 Les relations entre la capitale de la Monarchie et Zagreb étaient multiples et, en Croatie, on connaissait la modernité viennoise, alors que la littérature croate était beaucoup moins représentée dans les journaux viennois, comme par exemple dans le journal Zeit de Hermann Bahr, où les modernismes polonais ou tchèque étaient beaucoup plus présents.

22 Dans la littérature croate de la fin du siècle, on peut retrouver les mêmes tendances artistiques qu'à Vienne, mais sous des formes un peu moins diversifiées. Les textes de Vladimir Nazor (1876-1949) par exemple représentent le Jugendstil (le style «art nouveau ») et comportent en même temps des traits néoromantiques - si chers à la tradition croate. Dans le roman Albus roi, on retrouve une opposition claire entre la « simplicité ingénue » (Natürlichkeit), c'est-à-dire la vie « originelle », représentée dans 
la figure mythique du roi Albus, et l'artificiel (représenté par un arrangement ornemental des êtres et des choses) : tout l'entourage est soumis à la volonté humaine, c'est-à-dire à des lois artificielles. On retrouve donc les traits de la Sécession, mais on cherche en vain des motivations psychologiques aux événements présentés.

Comme dans l'art plastique, Nazor offre dans son roman une suite d'images statiques et décoratives dotées d'éléments symétriques: les figures incarnent certes la personnalisation des maximes esthétiques, mais il manque le lien caractéristique entre décadence et vitalisme. À la fin, le roi brutal de Nazor est vaincu par un principe opposé : celui d'un sentiment maternel mystique.

Un autre texte de la "moderna" croate, Die traurige Cascade und anderes, de 1913, évoque aussi la décadence; il s'agit d'un texte écrit en allemand par Tito Strozzi (1892-1970) qui représente la dialectique entre la vie et la mort, la vitalité et la déchéance. Suite aux reproches qu'on lui fit d'imiter le style viennois, Strozzi écrira ses textes ultérieurs en croate et changera aussi de style, abandonnant le Jugendstil (le style " art nouveau ») et se tournant vers une expression plus croate.

À côté d'eux, il existe aussi des écrivains « locaux » représentant la société patriarcale du village croate. Le plus connu est Josip Kosor (1879-1961) qui, par l'intermédiaire de Richard von Schaukal, de Stefan Zweig et de Hermann Bahr, connut une réception très favorable à Vienne. Cette réception positive peut s'expliquer par le fait que le public germanophone retrouva en lui le stéréotype des sujets rustiques, primitifs de la littérature croate (et d'autres pays slaves). On chercha dans son œuvre les traces d'un Tolstoï, d'un Dostoïevski ou d'un Gorki, ainsi que du vitalisme nietzschéen (comparable à Karl Schönherr, l'écrivain tyrolien).

On peut conclure en affirmant que les échanges et les transferts entre Vienne et Zagreb furent ambivalents et qu'il reste encore un champ de recherches très fructueux à défricher, notamment en matière d'échanges théâtraux (par exemple l'influence du Burgtheater), ainsi que dans les domaines de l'architecture et de la peinture.

\section{NOTES}

1. Spomenica u povodu proslave 300 godišnjice sveučilišta u Zagrebu, Discours à l'occasion du $300^{\mathrm{e}}$ Jubilé de l'Université de Zagreb, 1969, p. 336-340.

2. Voir F.Zenko, Aristotelizam od Petrića do Bošković, Zagreb 1983, surtout le chapitre «Recepcija Boškovićevich ideja na Neoacademia Zagrebiensis u 18.stoleću » («Réception des idées de Bošković dans la nouvelle académie de Zagreb au XVIII ${ }^{e}$ siècle »), p. 135-150.

3. Voir Erna Banić-Pajnić, Simeon Čučić 47 in : Prilozi za istražavanja hrvatske filozofske baštine (Contributions à la recherche de l'héritage philosophique croate), 1977/5-6, p. 79-103.

4. Zlatko Posavac, «Filozofia u Hrvatskoj 19. Stoleća » («Philosophie en Croatie du xIX siècle ») in : Praxis, 3/1967, p. 385-405.

5. Ivan Filipović, dans son essai «O samostalnosti škole» («Sur l'indépendance de l'école »), 1849, constate que l'indépendance de l'école par rapport à l'État était la condition de l'union « nationale » de la Yougoslavie. 
6. « Mlada Hrvatska » (Jeune Croatie) in : Mladost, I, 1898, n4 4, p. 177-187.

7. Das literarische Echo, II, 1900, p. 548-552.

\section{RÉSUMÉS}

Dans cette contribution, nous nous proposons d'analyser les relations entre Vienne et Zagreb dans trois domaines. Nous commencerons par étudier comment s'est opéré le transfert de la philosophie de l'université de Vienne vers la toute nouvelle université de Zagreb. Les étudiants de Zagreb qui ont étudié la philosophie à Vienne ont vivement souhaité importer dans leur pays tout ce qu'elle leur a inspiré, l'adapter à la mentalité croate, contribuant ainsi à renforcer le sentiment national croate et à affirmer une indépendance culturelle. Dans le domaine de la littérature, les échanges sont également marqués par une certaine sensibilité politique. Les traces qu'a laissées l'influence de la "modernité viennoise » dans l'évolution de l'art et la littérature croates sont très perceptibles. La fondation de la revue Mladost, s'inspirant de Ver Sacrum viennois n'est que l'un des nombreux exemples. Tandis que les revues de Zagreb se font régulièrement et avec enthousiasme l'écho de la vie culturelle viennoise, les publications viennoises parlent beaucoup moins de l'évolution de la vie culturelle et littéraire de Zagreb qu'elles ne le font pour la modernité tchèque et polonaise. Mais il existe aussi une résistance évidente à l'influence de la «décadence» viennoise, la «jeune» génération croate étant considérée comme génération de rêveurs et incarnant l'énergie perdue pour le jeune État croate. L'image de Vienne dans la littérature croate est très ambivalente. Vienne est représentée comme une ville attrayante, cosmopolite, mondaine, mais également comme une ville repoussante, dangereuse et destructrice.

In diesem Beitrag werden die Beziehungen zwischen Wien und Zagreb in drei Bereichen skizziert werden. Zunächst wird der Transfer der Philosophie der Wiener Universität an die neugegründete Universität in Zagreb untersucht. Die Zagreber Studenten haben die Inspirationen von ihrem Studium in Wien in dem Bestreben transferiert, sie der kroatischen Mentalität anzupassen und dadurch beizutragen, das kroatische Nationalbewusstsein zu stärken und der kulturellen Unabhängigkeit Ausdruck zu verleihen. Im Bereich der Literatur ist der Austausch ebenfalls von einer gewissen politischen Sensibilität gezeichnet. Der Einfluss der Wiener Moderne lässt einerseits deutliche Spuren in der Entwicklung der kroatischen Literatur und Kunst erkennen. Die Gründung der Zeitschrift „Mladost“ nach dem Vorbild von „Ver sacrum“ ist nur eines von vielen Beispielen. Während die Zagreber Zeitschriften regelmäßig oft begeistert über das kulturelle Leben in Wien berichten, wird in den Wiener Zeitschriften viel weniger über die Entwicklung in Zagreb berichtet als über die literarische Entwicklung der tschechischen oder der polnischen Moderne. Es gibt aber auch einen deutlichen Widerstand gegen den Einfluss der Wiener „Dekadenz“, die „junge“ kroatische Generation wird als Generation der Träumer angesehen und personifiziert die verlorene Energie für den jungen kroatischen Staat. Das Bild Wiens in der kroatischen Literatur ist ebenfalls sehr zweideutig. Einerseits wird Wien als anziehende, kosmopolitische, mondäne Stadt dargestellt, anderseits aber auch als abstoßend, gefährlich und zerstörerisch. 
INDEX

Mots-clés : Zagreb, Vienne

\section{AUTEUR}

\section{FRIDRUN RINNER}

Université de Provence, Aix-Marseille 